\title{
Editorial
}

\section{In search of a reliable marker of tissue injury during heart} surgery

Myocardial injury, manifested as transient cardiac contractile dysfunction ("stunning") and myocardial infarction, is the most frequent complication during heart surgery and is the single most important cause of hospital complications and death. Furthermore, patients who have a perioperative myocardial infarction have poor long term prognosis: only $51 \%$ of such patients remain free from adverse cardiac events after two years, compared with $96 \%$ of patients without infarction. ${ }^{1}$ Consequently, the detection of myocardial injury during cardiac surgery could be advantageous in the implementation of perioperative management, and it has long term prognostic implications.

Cardiac contractile dysfunction is the most prominent feature of myocardial injury despite the fact that there are virtually no perfect measures of postoperative cardiac function. The inotropic requirement is a "soft" measure and can vary considerably depending on the concomitant degree of volume administered, the depth of anaesthesia or postoperative sedation, and the particular guidelines for blood pressure and filling pressures regarded as acceptable in each hospital. Thermodilution techniques are load dependent and cannot estimate regional contractile impairment. Other invasive procedures such as conductance catheters and sonomicrometry provide a better assessment of the contractile state of the heart; however, they require invasive manipulation, which prevents their application clinically. Nuclear medicine methods, including magnetic resonance imaging and positron emission tomography, can evaluate tissue perfusion and metabolism in addition to global and regional contractility. Although there are obvious advantages in the simultaneous assessment of these functions, nuclear medicine tools are expensive, very few centres have access to the technology, and they are not applicable when the patient is on the operating table, soon after surgery or in patients in high dependency intensive care. Finally, the existing echo Doppler systems, although non-invasive and readily available, have the limitation of being sensitive to alterations in loading conditions, the interpretation is operator dependent and, at present, cannot yield an accurate assessment of contractile function.

Because of the numerous limitations with the assessment of cardiac contractile function, other methods to detect myocardial injury during cardiac surgery have been sought. ECG monitoring is constantly displayed for the first few hours after surgery and usually a full 12-lead ECG is only performed when myocardial injury is suspected. However, perioperative ECG changes are not indicative of reversible ischaemic injury, and ST-T wave changes are not reliable signs of myocardial infarction. New persistent $Q$ waves only can be considered as evidence of perioperative myocardial infarction. ${ }^{2}$ As a result, the assessment of a number of serum biochemical markers has been proposed to detect myocardial injury. It should be emphasised that many of these markers are released from damaged skeletal muscle as well as from the myocardium, and this lack of specificity makes them of limited use for the identification of myocardial injury.
However, some of the markers, including creatinine kinase (CK) MB isoenzyme and troponin T and I, are more specific and sensitive for cardiac damage, and they have been proposed as useful for diagnosing myocardial injury in daily clinical practice.

$\mathrm{CK}-\mathrm{MB}$ is the most commonly used biochemical marker to detect myocardial injury following cardiac surgery. Most patients undergoing cardiac surgery exhibit an increase in the release of $\mathrm{CK}-\mathrm{MB}^{13}$ even in the absence of other clinical and biochemical evidence of myocardial necrosis. Therefore, the threshold separating a routine perioperative increase from substantial damage is unclear, and the value and interpretation of this enzyme release remains to be defined. An additional problem is that the detection of CK-MB in plasma is not entirely specific for myocardial necrosis as skeletal muscle has a fourfold higher CK content than myocardium and it contains measurable CK-MB activity (2\%). ${ }^{4}$ Hence, the peak CK-MB increase may be of limited significance; it has been suggested that the pattern of CK-MB liberation from serial determinations may be more reliable for the detection of perioperative myocardial damage. ${ }^{15}$ Because of these problems, troponin $\mathrm{T}$ and $\mathrm{I}$ have been promoted as alternative markers of injury.

Troponins ( $\mathrm{T}$, I, and $\mathrm{C}$ ), which regulate muscle contraction through the tropomyosin complex, exist in cardiac and skeletal muscle; however, the isoforms troponin $\mathrm{T}$ and I have different protein structures and they are specific for the heart. ${ }^{6}$ It has been shown that both troponin $\mathrm{T}$ and I have a high sensitivity and specificity for the diagnosis of acute myocardial infarction. ${ }^{78}$ Detection of significant plasma release of cardiac troponin $\mathrm{T}$ and $\mathrm{I}$ has been observed previously in low risk patients undergoing myocardial revascularisation. ${ }^{9-12}$ In this issue, Koh and colleagues report on the utility of the assessment of troponin $\mathrm{T}$ for the determination of myocardial injury when measured in the coronary effluent of patients undergoing routine bypass graft surgery. ${ }^{13}$ They found that troponin $\mathrm{T}$ release was related to ischaemic time as well as to delayed recovery of left ventricular function and oxidative metabolism. These results confirm that troponin $\mathrm{T}$ can detect myocardial injury during bypass surgery, but more importantly, they show that the increased release of troponin $\mathrm{T}$ occurred in the presence of reversible myocardial damage.

The argument that the release of troponins may occur in stunned myocardium is supported by clinical findings that their plasma concentrations are raised in most patients undergoing cardiac surgery ${ }^{9-12}$ and by the demonstration that troponin $\mathrm{T}$ and other contractile proteins (for example, myosin, actin, and tropomyosin) exhibit complete disruption of their localisation pattern within 20 minutes of the onset of ischaemia ${ }^{14}$; a time period known to induce myocardial stunning in ischaemic cardiomyopathic human tissue. Further support for the hypothesis that troponin is degraded in the stunned myocardium is provided by the experimental demonstration that troponin degradation and the contractile dysfunction 
of stunning occur at the same time, and that both can be prevented by low $\mathrm{Ca}^{2+} /$ low $\mathrm{pH}$ reperfusion. ${ }^{15}$

Although Koh et al's report ${ }^{13}$ establishes an important relation between the release of troponin $\mathrm{T}$ and stunned myocardium in the human heart, their study does not disclose the threshold of troponin $\mathrm{T}$ release to determine whether any observed elevation represents reversible and therefore recoverable myocardium or, irreversible myocardium that is destined to die. This distinction is important for therapeutic decisions and prognostic implications.

Other aspects of Koh et al's study deserve attention. The measurement of troponin $\mathrm{T}$ release in the coronary effluent does not take into account enzyme release into the myocardial lymph and, by doing so, the peak and the total release of the protein may be underestimated. It is also worth noting that, because the half life of troponins is long (they can stay raised for at least four days after an acute myocardial infarction), ${ }^{16}$ the plasma increase detected during the early moments of reperfusion may not reflect the reperfusion injury sustained during a later phase or the presence of new ischaemic events that may occur during the postoperative period (such as acute occlusion of a coronary bypass graft).

There is consensus that a rapid and reliable test to ascertain the existence and degree of myocardial injury is much needed during cardiac surgery. This test will have the potential to aid in the selection of treatment strategies and it will provide a sensitive tool to investigate the effects of protective interventions. Troponin $\mathrm{T}$ and $\mathrm{I}$ appear to be good candidates; however, the following questions remain unanswered:

- What plasma concentration of these markers is required to establish the presence or absence of myocardial injury?

- Do plasma concentrations allow us to determine the extent of injury and permit a differential diagnosis between reversible and irreversible injury?

- Is a single plasma determination enough to detect any degree of myocardial injury?
- Alternatively, is the pattern and total amount of release of the marker required to grade the damage?

M GALIÑANES

Reader/Honorary Consultant in Cardiac Surgery,

University of Leicester,

The Glenfield Hospital, Groby Road,

Leicester LE3 9QP, UK

1 Guiteras Val P, Pelletier LC, Galinanes Hernandez M, et al. Diagnosis criteria and prognosis of perioperative myocardial infarction following coronary bypass. F Thorac Cardiovasc Surg 1983;86:878-86.

2 Brewer DL, Bilbro RH, Bartel AG. Myocardial infarction as a complication of coronary bypass surgery. Circulation 1973;47:58-64.

3 Griesmacher A, Grimm M, Schreiner W, et al. Diagnosis of perioperative myocardial infarction by considering relationship of postoperative electromyocardial infarction by considering relationship of postoperative electro-
cardiogram changes and enzyme increases after coronary bypass operacardiogram changes and enzym

4 Sylven C, Jansson E, Olin C. Human myocardial and skeletal muscle enzyme activities: creatine kinase and its isoenzyme $M B$ as related to citrate synthase and muscle fibre types. Clin Physiol 1983;3:461-8.

5 Delva E, Maillé JG, Solymoss BC, et al. Evaluation of myocardial damage during coronary artery grafting with serial determinations of serum. I Thorac Cardiovasc Surg 1978;75:467-75.

6 Katus HA, Remppis A, Scheffold T, et al. Intracellular compartmentation of cardiac troponin $\mathrm{T}$ and its release kinetics in patients with reperfused and nonreperfused myocardial infarction. Am f Cardiol 1991;67:1360-7.

7 Adams JE, Abendschein DR, Jaffe AS. Biochemical markers of myocardial injury: is MB creatine kinase the choice for the 1990s? Circulation 1993;88: $750-63$.

8 Katus HA, Remppis A, Neumann FJ, et al. Diagnostic efficiency of troponin $\mathrm{T}$ measurements in acute myocardial infarction. Circulation 1991;83:902-

9 Mair P, Mair J, Seibt I, et al. Cardiac troponin T: a new marker of myocardial tissue damage in bypass surgery. 7 Cardiol Vasc Anesth 1993;7:674-8.

10 Mair J, Larue C, Mair P, et al. Use of cardiac troponin I to diagnose perioperative myocardial infarction in coronary artery bypass grafting. Clin Chem 1994;40:2066-70.

11 Caputo M, Dihmis W, Birdi I, et al. Cardiac troponin T and troponin I release during coronary artery surgery using cold crystalloid and cold blood cardioplegia. Eur f Cardiothorac Surg 1997;12:254-60.

12 Jenkins DP, Pugsley WB, Alkhulaifi AM, et al. Ischaemic preconditioning reduces troponin $T$ release in patients undergoing coronary artery bypass surgery. Heart 1997;77:314-18.

13 Koh TW, Hooper J, Kemp M, et al. Intraoperative release of troponin T in coronary venous and arterial blood and its relation to recovery of left ventricular function and oxidative metabolism following coronary artery surgery. Heart 1998;80:341-8.

14 Hein S, Scheffold T, Schaper J. Ischemia induces early changes to cytoskeletal and contractile proteins in diseased human myocardium. $\mathcal{F}$ Thorac Cardiovasc Surg 1995;110:89-98.

15 Gao WD, Atar D, Liu Y, et al. Role of troponin I proteolysis in the pathogenesis of stunned myocardium. Circ Res 1997;80:393-9.

16 Mair J, Genser N, Morandell D, et al. Cardiac troponin I in the diagnosis of myocardial injury and infarction. Clin Chim Acta 1996;245:19-38.

\section{STAMPS IN CARDIOLOGY}

\section{Stethoscopes}

The stethoscope very occasionally appears in part of a medical stamp design and usually on stamps depicting some aspect of hypertension or blood pressure measurement. However, the 10 yen stamp from Japan in 1958 has the stethoscope as the central feature and was issued to commemorate the International Congresses of Chest Diseases and Broncho-

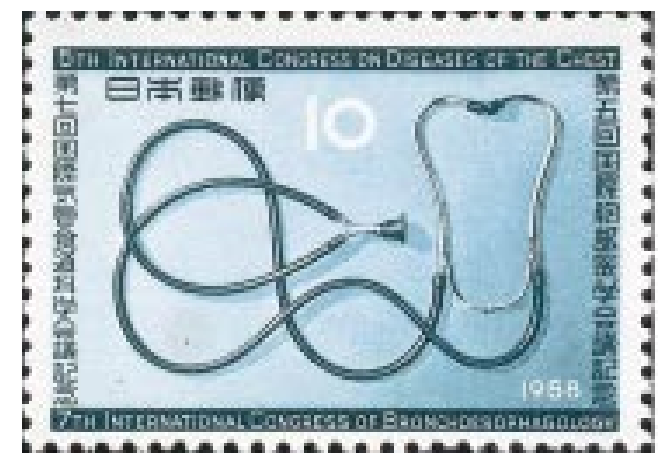

esophagology held in Tokyo. The stamp from Senegal is relatively rare being imperforate and was issued in April 1972 for the World Health Day. The stamp shows the World Health Organisation emblem, the ECG, and auscultation of the chest.

M K DAVIES A HOLLMAN

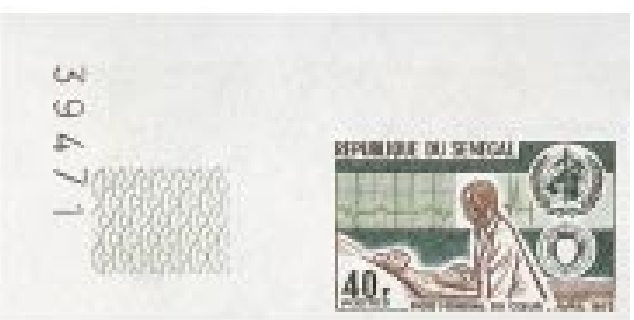

